\title{
Education in Global Health: Experience in Health-Promoting Schools Provides Trainees with Defined Core Competencies
}

\author{
Shreya Moodley, ${ }^{1}$ Arabat Kasangaki, ${ }^{2,3}$ and Andrew J. Macnab ${ }^{2,3}$ \\ ${ }^{1}$ Department of Pediatrics, The University of British Columbia, Vancouver, BC, Canada V6H 3 V4 \\ ${ }^{2}$ Makerere College of Health Sciences, Makerere University, P.O. Box 7072 Kampala, Uganda \\ ${ }^{3}$ Stellenbosch Institute for Advanced Study (STIAS), Wallenberg Research Centre, Stellenbosch University, Marais Street, \\ Stellenbosch 7600, South Africa \\ Correspondence should be addressed to Andrew J. Macnab, ajmacnab@gmail.com
}

Received 11 January 2012; Accepted 14 February 2012

Academic Editors: D. M. Hoffman, U. Moore, and M. Recker

Copyright () 2012 Shreya Moodley et al. This is an open access article distributed under the Creative Commons Attribution License, which permits unrestricted use, distribution, and reproduction in any medium, provided the original work is properly cited.

\begin{abstract}
Introduction. Medical education has defined essential "universal” core competencies. The value of global health education gained through participation in a health-promoting school project was assessed using Canada's CanMEDS roles and competencies. Methods. The project involved health care trainees in delivery of "Brighter Smiles," a global health education program addressing children's oral health in Canada and Uganda based on the WHO health-promoting (HP) school model. Multidisciplinary teams first visit a Canadian First Nations community for an introduction to HP schooling, team building, and experience working in different cultural environments and then have 4-6 weeks of global health project delivery in rural HP schools in Uganda in partnership with local College of Health Sciences trainees/faculty. Learning opportunities afforded were evaluated by conventional questionnaire and pilot categorization against the 7 CanMEDS roles (divided into 126 core competencies). Results. All collaborator and health Advocate competencies and 16/17 of the communicator roles were addressed. Overall, project experience included 88 (70\%) of the 126 competencies. Conclusions. This pilot suggests CanMEDS criteria can be used to effectively evaluate trainee participation in HP school program delivery, allowing the comprehensive educational opportunities to acquire global health knowledge and skills reported by conventional evaluation to be formally categorized against defined educational roles and competencies.
\end{abstract}

\section{Introduction}

Medical schools and Colleges of Health Sciences have acknowledged the need to educate their trainees to become "globally" competent and "well rounded" professionals. The CanMEDS Roles (2005), now recognized internationally, were developed by the Royal College of Physicians and Surgeons of Canada (RCPSC) to help Canadian medical schools instil the knowledge, skills, and attitudes necessary for modern, global practice amongst their trainees [1]. The RCPSC identified seven distinct, but overlapping, roles that health professionals need to have to function optimally in meeting the needs of a global society (see Figure 1):

(i) Medical expert/clinical decision-maker (the central role),

(ii) communicator, (iii) collaborator,

(iv) health advocate,

(v) manager,

(vi) scholar,

(vii) professional.

The CanMEDS roles have been integrated into the Royal College's accreditation standards, undergraduate health sciences curricula, objectives for postgraduate training, and final in-training evaluations. Each of these roles is further defined by "key competencies," which are further divided into "enabling competencies" that the practicing health professional will need to have and that trainees need to develop and demonstrate for qualification following residency training. However, some of these competencies are difficult to experience within the context of conventional 


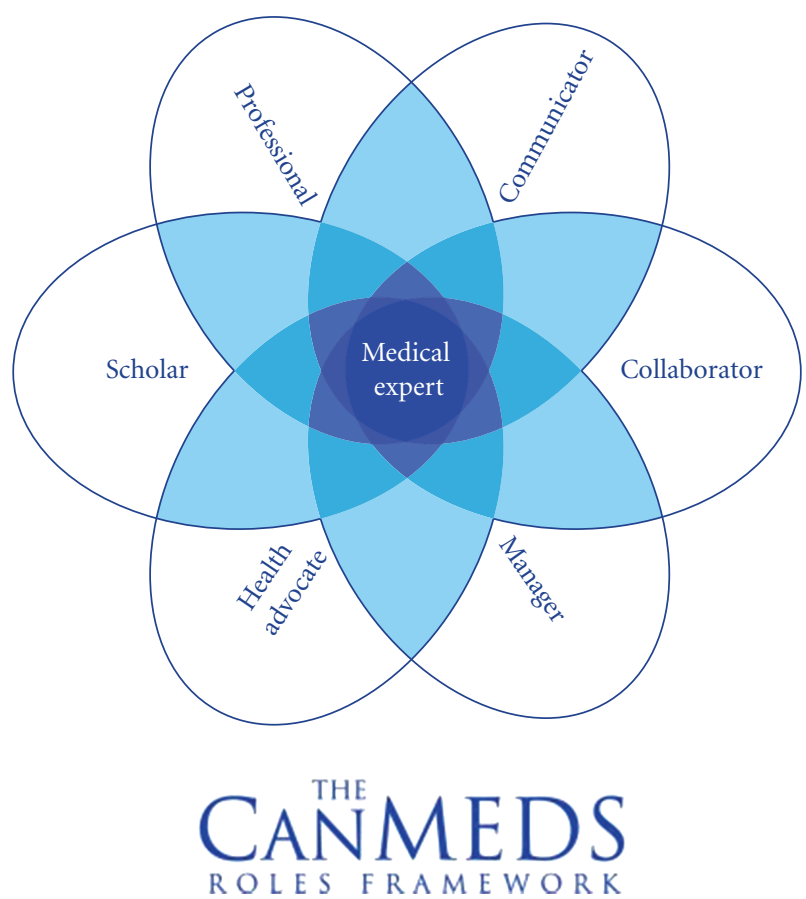

FIgURE 1

medical undergraduate and postgraduate health care training in developed countries.

With globalization, health care is no longer limited by geographical or political borders, making international health and social responsibility areas of importance during the training and future practice of health care professionals. Increased travel, trade, and communication between communities and countries have resulted in the spread and exchange of people, goods, knowledge, and disease. In addition, the increase and change in human migration have resulted in most developed countries having a very diverse population including immigrants and refugees with different health care risk factors and needs, making education that provides comprehensive knowledge and global health experience valuable.

In North America, both health care trainees and the universities where they receive their education have shown increased participation in global health, with reports in the literature of global health projects and program participation increasing by $25-50 \%$ over the last 10 years [2].

The term "global health" actually describes the concept that comprehensive health care involves addressing not only physiological health issues but also the broader determinants of health such as socioeconomic factors and access to health care. Consequently, "global health" issues are relevant everywhere, not just abroad. In 2000, the Brighter Smiles program began in British Columbia as a partnership established between a remote First Nations community and our university's pediatric residency training program $[3,4]$. The objectives were to promote oral health among aboriginal children using a "health promoting school" approach $[5,6]$ and to provide educational opportunities for medical trainees that were missing in their "urban" curriculum. This was achieved by a period of supervised immersion in a remote aboriginal community that enabled them to experience aboriginal culture and the challenges of remote health care delivery. In 2005, the Brighter Smiles program model program was "translated" for use in Uganda at the request of Makerere College of Health Sciences in Kampala as a means of establishing community-based learning opportunities for their students and as the first project in a collaborative partnership between Makerere University (MU) and the University of British Columbia (UBC).

Health-promoting schools (HPSs) as defined by the World Health Organization (WHO) use "a whole school approach to enhance the health and educational outcomes of children and adolescents through teaching and learning experiences initiated in the schools" $[5,6]$. HPSs incorporate health-related curriculum and promotion of healthy practices as part of each school day, and there is increasing recognition that involvement of health care trainees in HPS programs provides valuable learning opportunities with relevance to current objectives in global medical education [7].

This paper describes the use of a Canadian form of "universal" core competencies for medical education, the CanMeds criteria, to evaluate the value and relevance of global health education opportunities afforded to health care trainees by participation in delivery of a child health project based in health-promoting schools.

\section{Methods}

2.1. Project. "Brighter Smiles" is a version of the WHO HPS model $[5,6]$ that uses school-based oral health promotion as an entry point for school involvement. The program was begun in 2000 in a remote aboriginal community in Canada after extensive dialogue to establish a common goal and define an achievable health intervention of relevance to the community $[3,4]$. Trainees participating in delivery of this program work with their education faculty and members of the community to develop age and culturally appropriate health education modules that they deliver in class when they go to stay in the communities. Initially these were on the core topic of oral health. Subsequently the range of topics has expanded driven by collaborative discussion to address issues of interest and relevance to the community. When in the community trainees stay in family homes to establish rapport and experience the culture and patterns of daily life. In addition to teaching in class they promote the program daily healthy practice (tooth brushing by all pupils in a "brush in" held after lunch). Over time our trainees have been asked by the community to assume additional roles such as well child examinations or care of emergencies. Importantly, the confidence generated by success with the oral health intervention, and regular dialogue with the health care trainees, has enabled leaders in the communities to identify other health issues that they and the trainees have gone on to collaborate to address. Such issues have included low immunization rates, poor nutrition practices, 
the incidence of obesity, and prevalence of type 2 diabetes $[4,8]$. Thus, although oral health is the initiating focus, Brighter Smiles programs are intended to generate an interest in health literacy, engage the community, and build the capacity for health promotion to expand to include other issues relevant to the broader determinants of health [7]. By taking ownership of the program in this way partner communities drive the direction of the health promotion the program delivers and in doing so expose trainees to health delivery and cross-cultural issues relevant to the everyday challenges of health care in that community. This makes for vibrant and uniquely meaningful learning experiences for program participants, who are also involved in the evaluation processes essential to sustainability and effectiveness of global health programs.

"Brighter Smiles Africa" is modelled on the Canadian program and began in Uganda in 2005 as a collaborative partnership between the College of Health Sciences at Makerere University (MU), Kampala, and the Faculty of Medicine at the University of British Columbia (UBC) $[7,9]$. Joint MU/UBC teams have enrolled 5 communities. Initially the Brighter Smiles program was used to help establish community-based student learning opportunities that are now in place for all College of Health Sciences students. MU trainees visit participating schools throughout the year and are involved in similar roles and opportunities as their Canadian counterparts. Annually a team from UBC travels to Uganda to form an interdisciplinary team with MU of medical and dental faculty, students, and residents. This international interprofessional collaboration adds multiple dimensions to the regular component of project delivery and adds joint activities that include conduct of evaluation and collaborative research components. Prior to going to Uganda UBC teams are oriented to the philosophy and logistics of the Brighter Smiles HPS program during a stay in a Canadian partner community. In both the Canadian and Ugandan communities, collaboration involves interaction with local health care providers, school teachers, school principals, parents, community leaders, and administrators. Team members are able to participate in a range of activities involving the children that include examination of their oral health status, application of fluoride, promotion of healthy practices, and provision of education related to oral health, and now many other topics identified by each community. In addition they learn from interactions with team colleagues, immersion in another culture, experience of different health priorities and models of healthcare delivery, and techniques used for evaluation of the impact and effectiveness of the program $[7,9,10]$.

2.2. Participants. Two teams collaborate to deliver the "Brighter Smiles" program in Africa, one from the University of British Columbia (UBC), Canada and one from Makerere University (MU), Uganda, and each brings supervising faculty. In the year studied the UBC team had 5 Canadian health care students led by a pediatric second-year resident as team leader, and MU team involved 12 Ugandan dental students. The resident team leader's responsibilities included (i) advanced planning, administration, and project delivery,

(ii) collaborating and communicating with various local and international partners,

(iii) setting personal and team goals and objectives,

(iv) monitoring the health, welfare, and learning opportunities of team members,

(v) mentoring Canadian and Ugandan students,

(vi) organizing project logistics and supplies,

(vii) appropriately allocating funds.

2.3. Learning Opportunities. For all MU and UBC program participants these included

(i) travel to remote Canadian aboriginal and/or Ugandan communities,

(ii) immersion in unfamiliar environments and cultures,

(iii) collaborative interaction with aboriginal and international partners,

(iv) conduct of child oral examinations in the field setting,

(v) prophylactic application of topical fluoride,

(vi) exposure to unfamiliar health issues,

(vii) preparation and delivery of health education in class and for teachers,

(viii) participation in collaborative research (obtaining ethics and consent, data collection, instruction in methodology and design to Ugandan team),

(ix) project evaluation.

2.4. Data. The learning opportunities and experiences afforded by project participation were evaluated by all participants as in previous years using an anonymous selfassessment questionnaire [7]. UBC teams evaluated both their Canadian and Ugandan experiences and the MU team their collaborative experiences at Makerere and during project delivery in the rural communities. The resident team leader also documented her experiences daily, later contentcoded them against a table of the CanMEDS enabling competencies (as used in Canadian Medical schools) [1], and then further categorized by personal assessment whether each competency experienced was

(i) addressed during activities as a team leader,

(ii) addressed during delivery of defined elements of the project,

(iii) either unique to the global health experience or made different by it when compared to prior clinical experiences,

(iv) a directly CanMEDS-related experience or one that was more personal.

The results were summarized by counting the number of enabling competencies addressed in each of the 7 canMEDS "roles" and calculating the percentage of enabling competencies addressed. 
TABLE 1: The 7 CanMeds goals and key competencies [1]: documented during global health education in a health promoting school program.

\begin{tabular}{|c|c|c|c|c|c|}
\hline CanMEDS role & Key competency & As team leader & During program delivery & Exemplified by HPS & Total \\
\hline \multirow{6}{*}{ Expert } & Consultant & $1 / 7(14 \%)$ & $3 / 7(43 \%)$ & 0 & $3 / 7(43 \%)$ \\
\hline & Knowledge, skills & 0 & 0 & 0 & 0 \\
\hline & Assessment & 0 & $5 / 5100 \%$ & 0 & $5 / 5(100 \%)$ \\
\hline & Use interventions & $1 / 3(25 \%)$ & $3 / 4(75 \%)$ & 0 & $3 / 4(75 \%)$ \\
\hline & Procedures & 0 & $3 / 5(60 \%)$ & 0 & $3 / 5(60 \%)$ \\
\hline & Seek consultation & 0 & $3 / 3(100 \%)$ & 0 & $3 / 3(100 \%)$ \\
\hline \multirow{5}{*}{ Communicator } & Relationships with patients & $3 / 6(50 \%)$ & $6 / 6(100 \%)$ & $1 / 6(17 \%)$ & $6 / 6(100 \%)$ \\
\hline & Obtain information & 0 & $2 / 2(100 \%)$ & 0 & $2 / 2(100 \%)$ \\
\hline & Give info to patients & 0 & $1 / 1(100 \%)$ & 0 & $1 / 1(100 \%)$ \\
\hline & Develop common understanding & $1 / 5(20 \%)$ & $3 / 5(60 \%)$ & $2 / 5(40 \%)$ & $4 / 5(80 \%)$ \\
\hline & Oral and written report & 0 & $2 / 3(67 \%)$ & $1 / 3(33 \%)$ & $3 / 3(100 \%)$ \\
\hline \multirow{2}{*}{ Collaborator } & Interprofessional team & $6 / 10(60 \%)$ & $1 / 10(10 \%)$ & $10 / 10(100 \%)$ & $10 / 10(100 \%)$ \\
\hline & Interprofessional conflict & $3 / 6(50 \%)$ & 0 & $6 / 6(100 \%)$ & $6 / 6(100 \%)$ \\
\hline \multirow{4}{*}{ Manager } & $\begin{array}{l}\text { Contribute to organizational } \\
\text { effectiveness }\end{array}$ & $2 / 4(50 \%)$ & 0 & $3 / 4(75 \%)$ & $4 / 4(100 \%)$ \\
\hline & Manage practice and career & 0 & $3 / 4(25 \%)$ & 0 & $3 / 4(25 \%)$ \\
\hline & Allocate health care resources & $1 / 2(50 \%)$ & 0 & $2 / 2(100 \%)$ & $2 / 2(100 \%)$ \\
\hline & Administrative, leadership roles & $2 / 3(67 \%)$ & 0 & $2 / 3(67 \%)$ & $3 / 3(100 \%)$ \\
\hline \multirow{4}{*}{ Health advocate } & Respond to patient health needs & 0 & 0 & $3 / 3(100 \%)$ & $3 / 3(100 \%)$ \\
\hline & $\begin{array}{l}\text { Respond to community health } \\
\text { needs }\end{array}$ & 0 & 0 & $3 / 3(100 \%)$ & $3 / 3(100 \%)$ \\
\hline & Determinants of health & 0 & 0 & $2 / 2(100 \%)$ & $2 / 2(100 \%)$ \\
\hline & Promote health & $1 / 6(17 \%)$ & 0 & $3 / 6(50 \%)$ & $3 / 6(50 \%)$ \\
\hline \multirow{4}{*}{ Scholar } & Ongoing learning & 0 & 0 & $5 / 9(56 \%)$ & $5 / 9(56 \%)$ \\
\hline & Evaluate information and sources & 0 & 0 & 0 & 0 \\
\hline & Facilitate learning & $4 / 7(57 \%)$ & 0 & $2 / 7(29 \%)$ & $5 / 7(71 \%)$ \\
\hline & Contribute to new knowledge & 0 & 0 & $6 / 6(100 \%)$ & $6 / 6(100 \%)$ \\
\hline \multirow{3}{*}{ Professional } & Commitment & $1 / 6(17 \%)$ & $3 / 6(33 \%)$ & $2 / 6(50 \%)$ & $3 / 6(50 \%)$ \\
\hline & Participate in regulation & 0 & 0 & 0 & 0 \\
\hline & Commitment to physician health & 0 & 0 & 0 & 0 \\
\hline
\end{tabular}

\section{Results}

The learning opportunities and educational value trainees derive from participation in global health education delivery in health-promoting schools using the "Brighter Smiles" version of the WHO model have been reported previously, using data from conventional evaluation questionnaires and videotaped interviews $[7,9,10]$. Table 1 shows the tabulated results of the same opportunities and experiences categorized by the resident team leader using CanMEDS criteria. All of the enabling competencies of the collaborator and health advocate roles and 16 of the 17 (94\%) enabling competencies of the communicator role were addressed by participation in delivery of the Brighter Smiles program described. Overall, the experience covered 88 of the 126 (70\%) CanMEDS enabling competencies.

Table 1 shows the number and percent of enabling competencies within each key competency gained from participation as team leader, gained during global health program delivery, or exemplified by participation in delivery of the HPS program.

The Total column indicates how many of the enabling competencies were addressed in at least one way. (If an enabling competency was checked twice for example, experienced both as a team leader and during the elective, the Total column reflects only one count.)

The key competencies data in bold are those for which all (100\%) of enabling competencies were addressed.

Involvement in project delivery provided valuable and often unique opportunities. Relevant experiences related to CanMEDS covered all 7 roles; the highlights of these experiences in 14 of the enabling competencies are described below.

\subsection{Medical Expert}

3.1.1. Central Role. Gained pediatric clinical knowledge and skills acquisition beyond that regularly included in residency 
training in Canadian hospitals (including the impact of determinants of health, scope of health issues, range of diagnoses, severity of illness and mode of presentation, importance of prevention, variable availability of standard treatment options, impact of social factors on health and education, the relevance of cross-cultural issues, and burden of disease).

3.1.2. Key Competency 4: Use Preventive and Therapeutic Interventions Effectively. Developed skills in providing preventative interventions in rural communities and recognizing the impact of illness and injury prevention in a global health context.

\subsection{Communicator}

3.2.1. Key Competency 1: Develop Rapport, Trust, and Ethical Therapeutic Relationships with Patients and Families. Gained awareness of the cultural importance of family, role of family members in health-related decisions, and strength of extended family.

3.2.2. Enabling Competency 1.2: Establish Positive Therapeutic Relationships with Patients and Their Families That Are Characterized by Understanding, Trust, Respect, Honesty, and Empathy. Developed tools for effective communication with community leaders, parents, school staff, and children, since listening, dialogue, and mutual respect are fundamental to the success and sustainability of the Brighter Smiles partnership model. Learned novel approaches to some issues and different ways to address educational topics from Ugandan colleagues that altered my approach.

3.2.3. Enabling Competency 2.1: Gather Information about a Disease, and also about a Patient's Beliefs, Concerns, Expectations, and Illness Experience. Gained understanding of the challenges and opportunities faced when communicating in a different country, culture, or language. Had the importance of understanding local cultural beliefs, traditional practices, and medical realities when planning health interventions reinforced by the collaborative interactions experienced during this project.

\subsection{Collaborator}

3.3.1. Key Competency 1: Participate Effectively and Appropriately in an Interprofessional Healthcare Team. Developed teamwork skills that drew on the diverse knowledge of an interprofessional and international team working effectively in remote and unfamiliar locations and gained experience collaborating on a much larger scale than usual.

3.3.2. Enabling Competency 1.2: Describe the Roles and Responsibilities of Other Professionals within the Health Care Team. Practised multidisciplinary and multicultural approaches to health care; came to understand and make use of the unique perspectives of each team member and appreciate that collaboration brings new insights and experience that qualify existing knowledge, allow better planning and novel problem solving, and modify practice.

3.3.3. Enabling Competency 1.10: Where Appropriate, Demonstrate Leadership in a Healthcare Team. Learned and practiced new leadership skills to meet unexpected issues and unfamiliar goals while ensuring the health and "wellness" of individuals and my team.

\subsection{Manager}

3.4.1. Enabling Competency 2.2: Manage a Practice including Finances and Human Resources. Gained new familiarity with financial issues by administering funds for transport, accommodation, and supplies.

\subsection{Health Advocate}

3.5.1. Key Competency 1: Respond to Individual Patient Health Needs and Issues as Part of Patient Care. Gained experience in the conduct of needs assessments under field conditions with limited resources, in a manner responsive to unfamiliar cultural needs.

\subsubsection{Key Competency 3: Respond to Individual Patient Health} Needs and Issues as Part of Patient Care. Gained vivid awareness of the impact of broader determinants of health, poverty, and lack of basic health knowledge and also how to function with limited treatment options and understand the huge social impact and burden of disease that exists in Africa.

3.5.3. Key Competency 4: Promote the Health of Individual Patients, Communities, and Populations. Acquired insight into the physician's role as health advocate, incorporating the realization that, as global citizens, doctors have a powerful voice to promote health for the global community.

\subsection{Scholar}

3.6.1. Key Competency 3: Facilitate the Learning of Patients, Families, Students, Residents, Other Health Professionals, the Public, and Others, as Appropriate. Mentored Canadian and Ugandan medical and dental students; provided education to school children, parents, and teachers; shared medical knowledge with both local and international residents and faculty. Prepared, presented at scientific meetings, and published evaluation and research data from project participation.

3.6.2. Key Competency 4: Contribute to the Development, Dissemination, and Translation of New Knowledge and Practices. Gained an appreciation of the ethical issues related to research, essential nature of project evaluation for sustainability of global health projects, unique insights into the potential benefits and harms of international "aid" and negative impact of "medical tourism." 


\subsection{Professional}

3.7.1. Key Competency: Commitment. Adapted to immersion in new environments and new cultures, and gained unique understanding of the importance of relevant international health initiatives and effective North/South collaborative partnerships.

Came to recognize the similarities between the broader determinants of health for underserved populations at home and abroad and that individual communities respond to similar needs and challenges in different ways.

Provided service to the underserved in conditions of severe poverty, established a rapport and ongoing commitment to the international partners and rural communities encountered, and gained new perspective on the opportunities offered by a career as a practicing paediatrician in the context of global health.

\section{Discussion}

This study mirrors prior reports that global health learning experience gained by health care trainees through participation in health promoting schools projects provides a range of valuable educational opportunities $[7,9]$ and adds pilot data that such experience provides important opportunities to acquire or enhance CanMEDS skills and competencies. The experiences related to the CanMEDS roles of communicator, collaborator, and health advocate were particularly rich and formative, with lesser but still valuable opportunities afforded in the areas of medical expert/clinical decisionmaker, scholar, and manager. Some elements, especially those related to the role (health) professional, were gained in a manner that was sometimes unique and often more vivid than training opportunities provide in Canada. "Developing this understanding during my conventional residency rotations would have taken much longer; it would also have been less powerful and memorable, and hence less likely to impact my subsequent practice."

The value of international health electives (IHEs) and global health experience is becoming increasingly recognized particularly by North American universities. Individual reports suggest IHEs help make students better physicians, strengthen our medical system, and contribute to core competency [11]. Success in both launching and sustaining global health programs is more likely when universities work in true partnership with people and communities who know firsthand what their health concerns are and how best to address them $[12,13]$. IHEs have been shown to increase the probability that graduates will undertake careers in underserved or primary care settings [14], and also to affect positively participants' clinical skills, attitudes, and knowledge of tropical medicine $[15,16]$. Consequently, more global health training and opportunities, with greater support, have been called for in medical education $[17,18]$. However, justifying the time and expense of participating in an IHE or global health project can be difficult when the pressure to meet specific target objectives such as CanMEDS is so high. So it is important to evaluate which competencies can be developed during the educational opportunities such involvement affords and which are provided in a unique or more meaningful manner.

Health-promoting schools are a rich learning environment for health care trainees. While we go to HPS to educate and promote health, the children, adults, and situations we encounter in fact teach us too. With greater understanding of this paradigm, working to establish HPS could become a means of forging strategic partnerships between institutes of higher learning, the schools who become health promoting, and parallel agencies involved in health and education.

We recognize limitations in what we describe. This is predominantly a personal account and a pilot comparison of a single individual's experience and opportunities against the formal criteria of the CanMEDS roles and competencies. However, the results are mirrored by the written evaluations of the many trainees who have participated in the same program for many years, $[4,7,9]$ and unlike the descriptive (qualitative) nature of other published accounts of electives in international health, our data identifies that the CanMEDS criteria could potentially be used as a quantitative element to evaluate global health experience. Amongst the on-going development of evaluation tools for medical education, ones for evaluating the specific opportunities afforded by global health education during project delivery are known to be needed, hence the relevance of considering CanMEDS in the formal assessment of international health electives. The roles and competencies they encompass are internationally recognized and the criteria help medical schools instil the knowledge, skills, and attitudes necessary for modern global practice amongst their trainees, and formally asses that trainees become globally competent and "well rounded" professionals $[1,19]$. There is growing interest in the application of competency assessments across many environments and having reliable tools that can be used by a large number of assessors in different health care systems. A common exit interview or evaluation based on CanMEDS could be developed and trialled to address this need. Such a tool could be made available on line and ideally would become a requirement for all trainees participating in global health programs at home, or health electives abroad, to complete. Such data would aid health care trainees in the educational choices they make as they seek to become global citizens and guide the choices that must be made by faculty with the responsibility to make medical education globally relevant.

\section{Conclusion}

Participation in global health initiatives based on the WHO Health-Promoting School model by trainee health professionals has been shown to provide important opportunities and experience. This pilot study indicates the potential for the CanMEDS roles and competencies to be used to categorize the scope and impact of the education afforded by participation in such programs and suggests particular and often unique value in the context of the CanMEDS roles of communicator, collaborator and health advocate. 


\section{References}

[1] J. R. Frank, Ed., "CanMEDS 2005 Physician competency framework," 2005, http://meds.queensu.ca/medicine/obgyn /CanMEDS2005.booklet.pdf.

[2] B. D. Nelson, A. C. C. Lee, P. K. Newby, M. R. Chamberlin, and C. C. Huang, "Global health training in pediatric residency programs," Pediatrics, vol. 122, no. 1, pp. 28-33, 2008.

[3] A. J. Macnab, R. Rassekh, Y. H. Lai et al., "Development of a symbiotic relationship between a remote First nation's community leading to a school-based collaborative oral health program," IHS Primary Care Provider, vol. 30, no. 9, pp. 230233, 2005.

[4] A. J. Macnab, J. Rozmus, D. Benton, and F. A. Gagnon, "3-year results of a collaborative school-based oral health program in a remote First Nation's community," Rural and Remote Health, vol. 8, no. 2, p. 882, 2008.

[5] WHO, Nairobi Call to Action for Closing the Implementation Gap in Health Promotion, WHO, Geneva, Switzerland, 2009.

[6] L. St Leger, I. Young, C. Blanchard, and M. Perry, "Promoting health in schools from evidence to action," International Union for Health Promotion and Education, 2009, http://www.iuhpe.org/upload/Activities/Scientific _Affairs/CDC/PHiS-EtA.

[7] A. J. Macnab and A. Kasangaki, "Many voices, one song': Ugandan investment in child health using a health promoting school model," Health promotion International, vol. 27, no. 1, pp. 63-73, 2012.

[8] C. Panagiotopoulos, J. Rozmus, R. E. Gagnon, and A. J. Macnab, "Diabetes screening of children in a remote First Nations community on the west coast of Canada: challenges and solutions," Rural and Remote Health, vol. 7, no. 3, p. 771, 2007.

[9] A. J. Macnab, N. Radziminski, H. Budden et al., "Brighter Smiles Africa-translation of a Canadian community-based health-promoting school program to Uganda," Education for Health, vol. 23, no. 2, p. 241, 2010.

[10] A. J. Macnab, A. Kasangaki, and W. Canon, "Videotaped interviews as medium to enhance program evaluation," Health Promotion International, vol. 27, no. 1, pp. 74-81, 2012.

[11] M. E. Goecke, J. Kanashiro, P. Kyamanywa, and G. L. Hollaar, "Using CanMEDS to guide international health electives: an enriching experience in Uganda defined for a Canadian surgery resident," Canadian Journal of Surgery, vol. 51, no. 4, pp. 289-295, 2008.

[12] S. Moodley, R. M. Hurley, J. Druker, W. G. Cannon, A. J. Macnab, and A. Kasangaki, "Global health initiatives: value and relevancy to residency training," Journal of Investigative Medicine, vol. 57, no. 1, p. 239, 2009.

[13] J. P. Koplan and R. L. Baggett, "The emory global health institute: developing partnerships to improve health through research, training, and service," Academic Medicine, vol. 83, no. 2, pp. 128-133, 2008.

[14] A. J. Macnab, A. Kasangaki, and F. Gagnon, "Health promoting schools provide community-based learning opportunities conducive to careers in rural practice," International Journal of Family Medicine, vol. 2011, Article ID 892518, 5 pages, 2011.

[15] J. Kanashiro, G. Hollaar, B. Wright, K. Nammavongmixay, and S. Roff, "Setting priorities for teaching and learning: an innovative needs assessment for a new family medicine program in Lao PDR," Academic Medicine, vol. 82, no. 3, pp. 231-237, 2007.

[16] M. J. Thompson, M. K. Huntington, D. D. Hunt, L. E. Pinsky, and J. J. Brodie, "Educational effects of international health electives on U.S. and Canadian medical students and residents: a literature review," Academic Medicine, vol. 78, no. 3, pp. 342347, 2003.

[17] P. K. Drain, A. Primack, D. D. Hunt, W. W. Fawzi, K. K. Holmes, and P. Gardner, "Global health in medical education: a call for more training and opportunities," Academic Medicine, vol. 82, no. 3, pp. 226-230, 2007.

[18] P. K. Drain, K. K. Holmes, K. M. Skeff, T. L. Hall, and P. Gardner, "Global health training and international clinical rotations during residency: current status, needs, and opportunities," Academic Medicine, vol. 84, no. 3, pp. 320-325, 2009.

[19] J. R. Frank and D. Danoff, "The CanMEDS initiative: Implementing an outcomes-based framework of physician competencies," Medical Teacher, vol. 29, no. 7, pp. 642-647, 2007. 

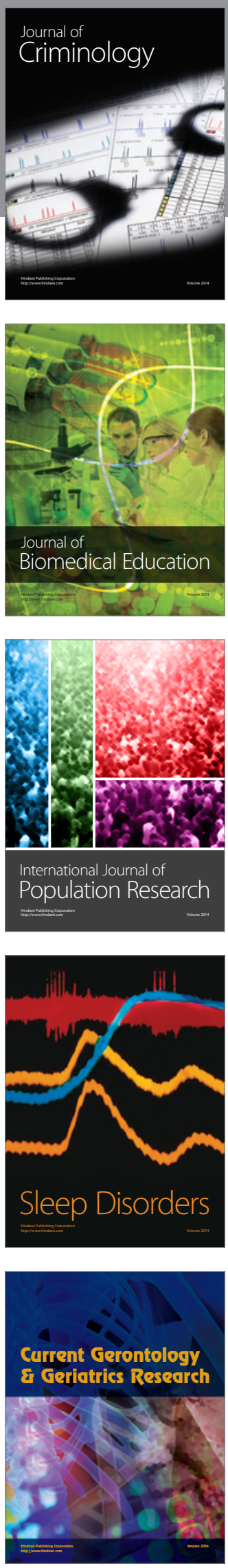
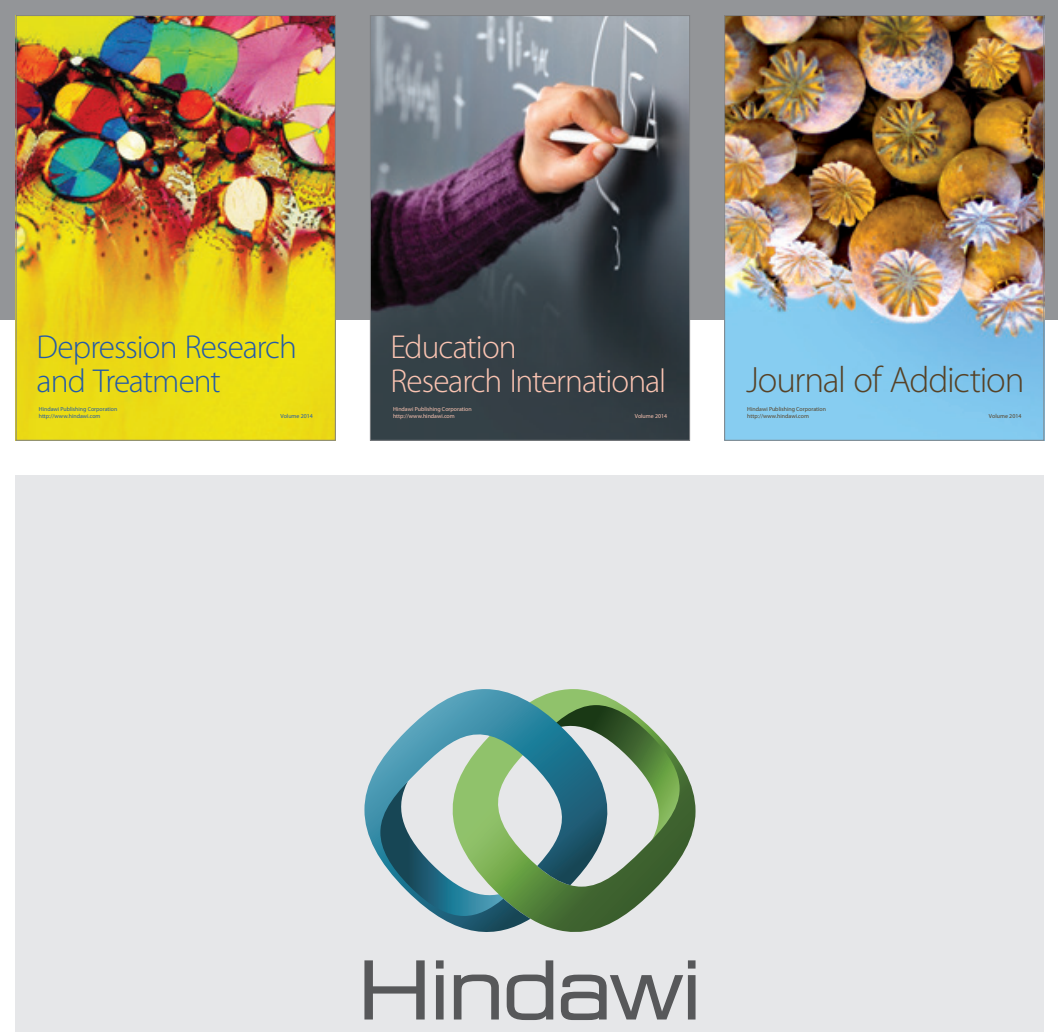

Submit your manuscripts at

http://www.hindawi.com

Child Development Research
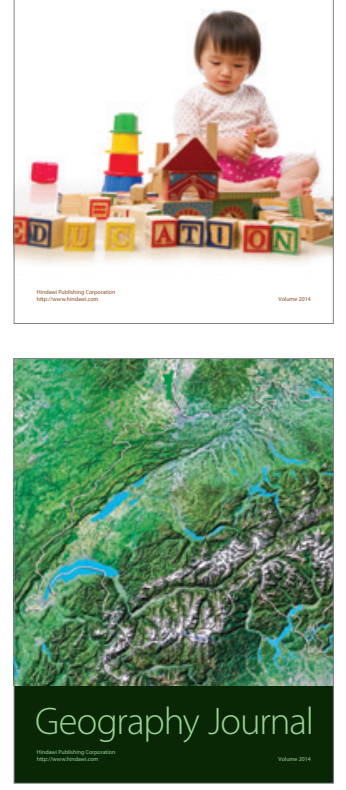

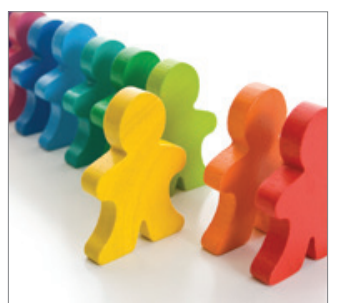

Autism

Research and Treatment
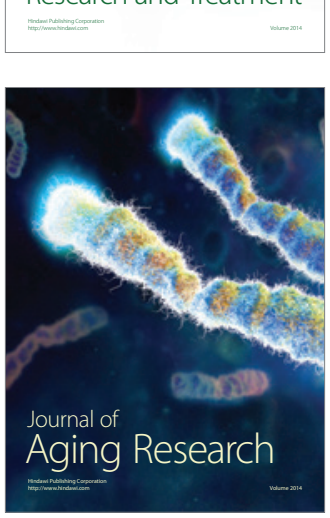
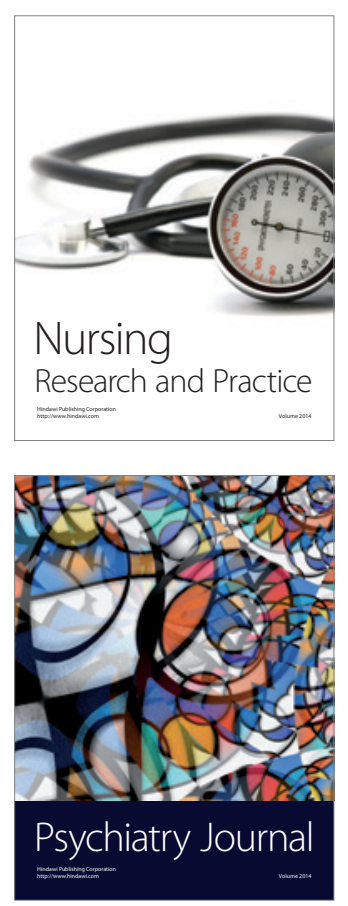
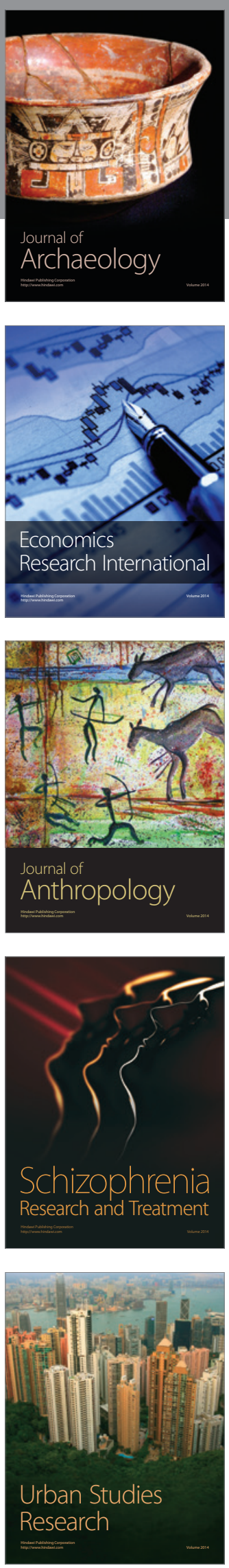